to a healthy control group $(7.6 \%$ versus $5.85 \%, p=0.129)$. After a 12 week treatment with alfacalcidol in patients with RA, an increase in the percentage of activated Treg cells (HLA-DR + relative to total T-reg lymphocytes) was observed in relation to the values detected at the beginning of the study, up to the level recorded in the group of healthy controls $6.13 \%$ vs. $4.76 \%, p=0.219$ ).

Conclusion: It is believed that functional blockade of Treg cells plays the most important role in RA immunopathogenesis, most likely due to inhibition of their function by proinflammatory cytokines, due to an increase in the number of activated effector $\mathrm{T}$ cells, or perhaps due to the fact that some completely differentiated $T$ reg cells can be very unstable. Our results are in favor of the potential immunomodulatory effect of alfacalcidol in autoimune Th1/Th17-mediated diseases.

Disclosure of Interests: Tatjana Radnic: None declared, Katarina Pasalic: None declared, Mirjana Sefik Bukilica: None declared, Ivanka Markovic: None declared, Nemanja Damjanov Grant/research support from: AbbVie Pfizer and Roche, Consultant for: Abbvie, Gedeon Richter, Merck, Novartis, Pfizer and Roche., Speakers bureau: Abbvie, Gedeon Richter, Merck Novartis, Pfizer and Roche., Jelena Vojinovic: None declared DOI: 10.1136/annrheumdis-2019-eular.6048

\section{SAT0029 \\ THE IMMUNE-PATHOGENIC CHARACTERISTICS OF AUTOREACTIVE B CELLS AGAINST CITRULLINATED ANTIGENS IN RHEUMATOID ARTHRITIS}

Hendy Kristyanto ${ }^{1}$, Ellen van der Voort ${ }^{1}$, Priscilla Kerkman ${ }^{2}$, Leonie Burgers ${ }^{1}$, Robin Ten Brinck ${ }^{1}$, Annette van der Helm - van Mil $^{1}$, Dominique Baeten ${ }^{3,4}$, Thomas Huizinga ${ }^{1}$, Rene Toes ${ }^{1}$, Hans Ulrich Scherer ${ }^{1} .{ }^{1}$ Leiden University Medical Center, Department of Rheumatology, Leiden, Netherlands; ${ }^{2}$ University Medical Center Utrecht, Department of Medical Microbiology, Utrecht, Netherlands; ${ }^{3}$ Academic Medical Centre, Department of Clinical Immunology and Rheumatology, Amsterdam, Netherlands; ${ }^{4}$ UCB Pharma, Brussels, Belgium

Background: Autoreactive B cells are critical mediators of autoimmune pathology. At present, little is known about the functional and phenotypic characteristics of these cells in human autoimmune disease. Rheumatoid arthritis (RA), a common autoimmune disease, is characterized by the presence of disease-specific anti-citrullinated protein antibodies (ACPA) Different lines of evidence indicate that CD20+ B cells, and in particular the citrullinated antigen-specific, ACPA-expressing subset, are critically involved in disease pathogenesis.

Objectives: To delineate the molecular make-up of ACPA-expressing B cells and to define their pathogenic effector functions.

Methods: Protective tetanus toxoid (TT)-specific and autoreactive ACPAexpressing $B$ cells were identified and analyzed directly ex-vivo from individual RA patients and from ACPA-positive individuals at-risk for developing disease. Both antigen-specific cell populations were enumerated and phenotypically characterized by flow cytometry. In addition, ACPA-expressing B cells were isolated and functionally analyzed in B cell culture systems.

Results: In contrast to TT-specific B cells from the same patients, ACPAexpressing $B$ cells were larger in size and strongly expressed CD19, HLA-DR, CD80, CD86 and the proliferation marker Ki-67, while down-reg ulating CD32. This activated phenotype was less pronounced in ACPA positive 'at-risk' arthralgia patients. Furthermore, ACPA-expressing B cells in blood of RA patients secreted elevated levels of pro-inflammatory cytokines, in particular IL-8, upon stimulation and did so spontaneously as differentiated plasmablasts/-cells in synovial fluid. Notably, ACPA-expressing B cell-derived IL-8 induced neutrophil migration in vitro.

Conclusion: Our findings provide first evidence that ACPA-expressing B cells are well equipped to activate $T$ cells, actively differentiate into IL-8 producing plasmablasts and, hence, could attract neutrophils to the rheumatoid joint. These findings define important phenotypic and functional characteristics of autoreactive B cells in a prototypic human autoimmune disease. They point to a direct pathogenic role of ACPA-expressing B cells in the inflammatory disease process underlying RA and favour approaches that aim at their antigen-specific depletion.

Disclosure of Interests: Hendy Kristyanto: None declared, Ellen van der Voort: None declared, Priscilla Kerkman: None declared, Leonie Burgers: None declared, Robin ten Brinck: None declared, Annette van der Helm - van Mil Grant/research support from: The research leading to these results has received funding from the European Research Council (ERC) under the European Union's Horizon 2020 research and innovation programme (Starting grant, agreement No 714312) and from the Dutch Arthritis Foundation. The funding source had no role in the design and conduct of the study., Dominique Baeten Employee of: UCB Pharma, Thomas Huizinga Consultant for: Merck, UCB, Bristol Myers Squibb, Biotest AG, Pfizer, GSK, Novartis, Roche, Sanofi-Aventis, Abbott, Crescendo Bioscience Inc., Nycomed, Boeringher, Takeda, Zydus, Epirus, Eli Lilly, Rene Toes Grant/research support from: Sanofi, Hans Ulrich Scherer Grant/research support from: Sanofi, BMS

DOI: 10.1136/annrheumdis-2019-eular.6409

\section{SAT0030 CITRULLINE-REACTIVE B CELLS ARE PRESENT IN INFLAMED GINGIVAL TISSUE AND DISPLAY CROSS- REACTIVITY BETWEEN BACTERIAL AND HUMAN ANTIGENS}

Natalia Sherina ${ }^{1}$, Vijay Joshua ${ }^{1}$, Radha Thyagarajan ${ }^{1}$, Natalie Sippl $^{1}$

Lena Israelsson ${ }^{1}$, Heidi Wähämaa', Ragnhild Stålesen ${ }^{1}$, Kaja Eriksson ${ }^{2}$,

Tülay Yucel-Lindberg ${ }^{2}$, Aase Hensvold ${ }^{1}$, Caroline Grönwall' ${ }^{1}$, Anca Catrina ${ }^{1}$

Vivianne Malmström ${ }^{1}$, Antonio Lanzavecchia ${ }^{3}$, Luca Piccoli ${ }^{3}$, Khaled Amara ${ }^{1}$

Karin Lundberg'. ${ }^{1}$ Karolinska Institute, Department of Medicine, Stockholm,

Sweden, ${ }^{2}$ Karolinska Institutet, Department of Dental Medicine, Stockholm,

Sweden; ${ }^{3}$ Università della Svizzera Italiana, Institute for Research in Biomedicine

Bellinzona, Switzerland

Background: Antibodies targeting citrullinated proteins (ACPA) are highly specific for rheumatoid arthritis (RA). However, the etiology and molecular basis for ACPA production is still unclear. Based on an epidemiological association between RA and periodontitis (PD), and the unique ability of the oral pathogen P.gingivalis $(P g)$ to express a PAD enzyme that can citrullinate both bacterial and human proteins, it has been hypothesised that the ACPA response may be triggered in the gum mucosa in response to $\mathrm{Pg}$.

Objectives: The main purpose of this study was to investigate if citrulline-reactive $B$ cells reside in inflamed gingival tissue, and to examine ACPA cross-reactivity between citrullinated bacterial and human epitopes on a monoclonal level.

Methods: Using a single-cell antibody cloning approach, 55 recombinant monoclonal antibodies (mAbs) were generated from gingival tissue (GT) CD19+ $B$ cells ( $n=1$ ACPA+ RA/PD patient). Citrulline reactivity was determined using the anti-CCP2 kit (EuroDiagnostica $A B$ ), and in-house peptide ELISAs (including a citrullinated peptide derived from $P g$ PAD (CPP3) and citrullinated peptides derived from human $\alpha$-enolase, fibrinogen, vimentin, fillaggrin and histone 4). Reactivity against CCP2 and CPP3 was also investigated in: 19 mAbs from bronchoalveolar lavage (BAL) CD19+ B cells ( $\mathrm{n}=2$ ACPA+ RA patients); 29 mAbs from bone marrow (BM) plasma cells ( $n=4$ ACPA+ RA patients); 142 mAbs from synovial fluid (SF) plasma cells ( $n=5$ ACPA+ RA patients); and 36 mAbs from peripheral blood memory/plasma cells ( $n=4$ ACPA+ RA patients). Predicted germline versions were produced for two of the mAbs.

Results: Among 55 GT mAbs, 14 unique clones (25\%) were reactive to the bacterial CPP3-peptide. We also detected CPP3-reactive mAbs from BAL $(n=9 / 19)$, BM $(n=3 / 29)$, SF $(n=1 / 142)$ and peripheral blood $(n=1 / 36)$. Interestingly, 11 out of 28 (39\%) CPP3-reactive clones also bound citrullinated peptides derived from human proteins. Notably, three of these clones were positive in the clinical anti-CCP2 test, and when converted back to the predicted germline sequence, these clones became CCP2 negative, while maintaining reactivity against the bacterial CPP3 peptide. Conclusion: Our data show that $B$ cells reactive with a citrullinated peptide derived from $P g$ PAD are present in gingival tissue, lungs, bone marrow, blood and the inflamed joint of ACPA+ RA patients. Moreover, the finding that a number of these clones are cross-reactive with citrullinated peptides derived from human proteins as well as the gold standard CCP2 test suggests mechanisms of molecular mimicry in the generation of ACPA. Importantly, the germline versions of these ACPA were $\mathrm{Pg}$ reactive but not autoreactive, supporting the hypothesis of a bacterial origin for this ACPA response.

Disclosure of Interests: Natalia Sherina: None declared, Vijay Joshua: None declared, Radha Thyagarajan: None declared, Natalie Sippl: None declared, Lena Israelsson: None declared, Heidi Wähämaa: None declared, Ragnhild Stålesen: None declared, Kaja Eriksson: None declared, Tülay Yucel-Lindberg: None declared, Aase Hensvold: None declared, Caroline Grönwall: None declared, Anca Catrina Grant/research support from: Yes, but not for the presented study., Vivianne Malmström: None declared, Antonio Lanzavecchia: None declared, Luca Piccoli: None declared, Khaled Amara: None declared, Karin Lundberg: None declared DOI: 10.1136/annrheumdis-2019-eular.6278 